\title{
Musicoterapia no atendimento domiciliar: avaliações para saúde e bem-estar de idosos
}

\author{
Rafaela Santos Soares \\ Pesquisadora independente \\ rafaelamusicoterapeuta@gmail.com \\ Marina Horta Freire \\ Universidade Federal de Minas Gerais. Escola de Música \\ marinahf@gmail.com
}

\begin{abstract}
Resumo: Este artigo apresenta os resultados iniciais de pesquisa na área de Musicoterapia, realizadas com idosos em atendimento domiciliar. Visando observar os possíveis benefícios da Musicoterapia, participaram 33 idosos que recebiam atendimento da instituição parceira, divididos aleatoriamente em grupo Intervenção e Controle. Os idosos do grupo Intervenção participaram de programa de 10 sessões musicoterapêuticas no intervalo de 21 semanas e tiveram apoio de vídeos complementares com material pensado individualmente. Os idosos do grupo Controle receberam seus cuidados habituais sem as sessões musicoterapêuticas nem os vídeos de apoio. As avaliação inicial e final foram feitas de forma cega por meio de material audiovisual gravado, utilizando a escala SEMPA (INGELMO, 2012) para avaliar as dimensões psicomotora, perceptivo-cognitiva, comunicacional e socioemocional dos participantes. Os resultados demonstraram que as avaliações iniciais sempre foram melhores que as finais em ambos os grupos designados. Futuras análises, com outras escalas, serão feitas para a continuação desta pesquisa e verificação dos resultados iniciais.
\end{abstract}

Palavras-chave: Musicoterapia, Idosos, Atendimento domiciliar, Avaliação.

\section{Music therapy in home care: assessments for the health and well-being of the elderly}

\begin{abstract}
This article shows the initial results of a Music therapy research, conducted with elder people in a home care setting. Aiming to observe the possible benefits from Music therapy, 33 people who received care from a partner institution were randomly separated into a Control and Intervention group. The Intervention group participated of a 10 sessions Music Therapy program, during 21 weeks, and had access to supporting videos, which had person-specific content. The Control group had no Music Therapy session but received their standard care. The initial and final assessments were done in a blind experiment through recording, using the SEMPA scale (INGELMO, 2012) in order to evaluate the psychomotor, perceptual-cognitive, communicational and socioemotional dimensions of the participants. The results showed that the initial assessments were always superior in both groups. Future analysis, with other scales, will be made to the ongoing research, along with a verification of the initial results.
\end{abstract}

Keywords: Music therapy, Elderly, Home care, Assessment.

\section{Introdução}

O presente estudo apresenta os resultados iniciais de uma pesquisa realizada por integrantes do corpo docente e discente da Universidade Federal de Minas Gerais (UFMG) em parceria com a empresa de cuidados extra médicos CAPTAMED Cuidados Continuados Ltda. e seus colaboradores. O objetivo geral da pesquisa é verificar evidências de que a utilização da Musicoterapia pode auxiliar e melhorar a saúde e bem-estar dos idosos atendidos em domicílio. 
De acordo com a Federação Mundial de Musicoterapia (WFMT, 2011), a Musicoterapia pode ser conceituada como a utilização da música e de seus elementos em intervenções terapêuticas conduzidas por profissional musicoterapeuta qualificado, em diversos contextos de saúde, de educação e contextos sociais, com indivíduos, grupos, famílias e/ou comunidades, "que procuram melhorar o seu bem-estar físico, social, comunicativo, emocional, intelectual, espiritual e a sua qualidade de vida".

$\mathrm{O}$ atendimento domiciliar ainda é pouco estudado na literatura musicoterapêutica, tendo sido apontado por Barcellos (2014) como uma nova e ascendente prática em Musicoterapia. De acordo com Karst (2015), o atendimento domiciliar transcende os limites do ambiente clínico/hospitalar, e realça a importância das intervenções multiprofissionais que visem contribuir com o aumento de autonomia e qualidade de vida em serviços humanizados que levam em consideração o indivíduo, a família e os contextos em que vivem.

O público-alvo deste estudo é a população idosa, que é crescente no Brasil, para a qual os tratamentos de saúde tentam minimizar perdas e enfrentar os impactos motores, cognitivos, sociais e psicológicos, naturais ou patológicos, do envelhecimento (PINHEIRO et al., 2017). Segundo Pinheiro e colaboradores (2017), a Musicoterapia pode exercer papel importante na redução desses impactos e aumento da qualidade de vida do idoso. Para apresentar a fundamentação musicoterapêutica com esse público, apontamos a seguir os principais benefícios da Musicoterapia nas quatro principais dimensões do idoso: psicomotor, perceptivocognitiva, comunicacional e socioemocional. Essas dimensões são também o escopo da escala SEMPA (Sistema de Evaluación Musicoterapéutica para Personas con Alzheimer), que foi utilizada na presente pesquisa e será descrita mais adiante neste estudo.

Ingelmo (2012, p. 101) afirma que o desenvolvimento psicomotor deve ser conceituado como "a interação mútua e dinâmica entre os esquemas motores, cognitivos e afetivos", e não somente como a manifestação progressiva de habilidades motoras no decorrer do tempo. Considerando essa informação e pesquisas atuais na área, é possível afirmar que a Musicoterapia é capaz de melhorar aspectos psicomotores como a extensão do movimento, as funções cardiorrespiratórias, a coordenação, o equilíbrio e marcha, reforçar a estimulação sensorial e promover estados de relaxamento, aliviando o estresse e proporcionando qualidade de vida (CEBA et al, 2012).

No que diz respeito à cognição, esse é o resultado do "funcionamento global das diferentes funções psicológicas, como percepção, orientação, atenção/concentração, memória 
de curto e longo prazo, comunicação, linguagem, resolução de problemas, etc." (INGELMO, 2012, p. 132). De acordo com Ceba e colaboradores (2012, p. 358) pode-se promover a melhora de todas essas funções cognitivas através das atividades musicoterapêuticas: "melhora a retenção de informação, estimula a memória de longo e curto prazo e a motivação, melhora atenção e orientação espaço temporal da pessoa em sua realidade".

Em relação à comunicação humana, seu processo é dinâmico e bidirecional, podendo se manifestar de forma verbal e não verbal (INGELMO, 2012). Além disso, a comunicação precede a linguagem, tanto para o indivíduo como para a espécie humana. A linguagem verbal complexa e articulada tem suas origens filo e ontogenéticas na comunicação não verbal, realizada por meio de gestos não convencionais, expressões faceais e a musicalidade da voz (andamento, dinâmica e entonação) (INGELMO, 2012). Associando este conceito aos idosos com demências, Sequera-Martín e colaboradores (2015, p. 94) dizem que a "prosódia e a sintaxe, afetadas nos estágios iniciais da Doença de Alzheimer, são aspectos compartilhados pela linguagem e pela música, essenciais para entender o significado e o valor emocional de uma mensagem ou conversa." (Tradução nossa).

A socialização e humor também são importantes dimensões da vida do idoso. Um estudo qualitativo realizado por Coll (2014) convidou idosos a realizarem alguma atividade musical a recordar sua biografia musical através de entrevistas, relacionando isso, por fim, com o envelhecimento ativo.

Os resultados desta análise confirmam o poder da bibliografia musical como ferramenta para a reminiscência, uma vez que recordar das experiências vividas com a música implica expressar muitas outras vivências/emoções importantes da sua história (COLL, 2014, p. 137).

Marques (2011) afirma que ao estimular o contato do idoso com sua identidade, relembrando sua história de vida e fortalecendo a autoestima, a Musicoterapia auxilia no envelhecimento ativo enquanto trabalha nas funções cognitivas do idoso, considerando-a assim uma terapia auto expressiva. Segundo Sequera-Martín et al (2015), a música proporciona uma resposta neurológica integrativa e global. Através dela é gerada uma intensa resposta emocional que está carregada de significado e depende de diferentes processos psicológicos.

Visto a importância da música e da Musicoterapia na saúde e na qualidade de vida do idoso, o presente estudo investiga as mudanças e benefícios que a Musicoterapia domiciliar pode trazer para a saúde dessa população. 


\section{Metodologia ${ }^{1}$}

Foram selecionados 50 idosos, que são atendidos pela instituição parceira da pesquisa, e divididos aleatoriamente em dois grupos: Intervenção e Controle. O grupo controle apenas participou das sessões de avaliação Inicial e Final, enquanto o grupo Intervenção recebeu ativamente as sessões musicoterapêuticas presenciais e acompanhamentos através de material de apoio musicoterapêutico. As avaliações do grupo Intervenção e Controle foram feitas no mesmo espaço de tempo de 21 semanas. Os participantes de ambos os grupos mantiveram seus cuidados habituais fornecidos pela mesma instituição. A caracterização dos grupos foi feita por meio da idade e dos diagnósticos dos participantes.

Os idosos do grupo Intervenção receberam atendimento musicoterapêutico domiciliar através do convênio realizado com a empresa CAPTAMED. O programa consistia em 10 sessões com duração de 50 minutos, sendo elas individuais e realizadas com o seguinte espaçamento de tempo: as 4 primeiras sessões semanalmente, as 3 seguintes quinzenalmente e as 3 últimas mensalmente. Algumas sessões foram realizadas por uma musicoterapeuta graduada, denominada de musicoterapeuta principal, e outras sessões foram realizadas por graduandos em Musicoterapia, denominados de musicoterapeutas assistentes, supervisionados pela musicoterapeuta principal. Na primeira sessão ( $1^{\mathrm{a}}$ semana) foi realizada a avaliação (igual à avaliação realizada com o grupo controle) sendo seguida por duas sessões semanais com os musicoterapeutas assistentes.

$\mathrm{Na}$ quarta sessão ( $4^{\mathrm{a}}$ semana), quando os atendimentos começaram a ser quinzenais, foi introduzido e explicado o material de apoio, que consiste em vídeos com atividades musicais, conduzidas pela própria musicoterapeuta principal, para serem feitas em casa nas semanas em que não haveria sessão musicoterapêutica - vídeos esses que não substituem a presença do terapeuta, mas são pensados de acordo com as necessidades já observadas durante as sessões anteriores. Na $5^{\mathrm{a}}$ e $6^{\mathrm{a}}$ sessão ( $6^{\mathrm{a}}$ e $8^{\mathrm{a}}$ semana) o processo continuou sendo conduzido pelo musicoterapeuta assistente, quinzenalmente. A $7^{\mathrm{a}}$ sessão $\left(10^{\mathrm{a}}\right.$ semana $)$ foi conduzida pela musicoterapeuta principal, seguida por duas sessões mensais com os assistentes. $\mathrm{O}$ encerramento foi feito pela musicoterapeuta principal através de uma avaliação final na $10^{\mathrm{a}}$ sessão ( $21^{\mathrm{a}}$ semana) (igual à avaliação realizada com o grupo controle). Esse cronograma de organização das sessões pode ser observado no Quadro 1, abaixo:

\footnotetext{
${ }^{1}$ Essa pesquisa foi aprovada pelos Comitês de Ética em Pesquisa da CAPTAMED Cuidados Continuados Ltda e da Universidade Federal de Minas Gerais (UFMG), sob o número CAAE 71177717.2.0000.5149, e pode ser consultado na Plataforma Brasil: < https://plataformabrasil.saude.gov.br/login.jsf $>$.
} 
Quadro 1 - Organização das sessões de Musicoterapia.

\begin{tabular}{c|l|l|l|l|l|l|l|l|l|l}
\hline SESSÃO & 1 & 2 & 3 & 4 & 5 & 6 & 7 & 8 & 9 & 10 \\
\hline SEMANA & $1^{\mathrm{a}}$ & $2^{\mathrm{a}}$ & $3^{\mathrm{a}}$ & $4^{\mathrm{a}}$ & $6^{\mathrm{a}}$ & $8^{\mathrm{a}}$ & $10^{\mathrm{a}}$ & $13^{\mathrm{a}}$ & $17^{\mathrm{a}}$ & $21^{\mathrm{a}}$ \\
\hline MUSICOTERAPEUTA & $\mathrm{P}$ & $\mathrm{A}$ & $\mathrm{A}$ & $\mathrm{P}$ & $\mathrm{A}$ & $\mathrm{A}$ & $\mathrm{P}$ & $\mathrm{A}$ & $\mathrm{A}$ & $\mathrm{P}$ \\
\hline
\end{tabular}

Elaboração da Autora. Legenda: P - Principal / A - Assistente.

As sessões de avaliações iniciais e finais foram conduzidas e gravadas (áudio e vídeo) pela musicoterapeuta principal, e avaliadas por uma pesquisadora que não participou dos atendimentos. A avaliação foi cega, ou seja, a pesquisadora não sabia a qual grupo pertencia cada idoso.

O protocolo utilizado para as avaliações foi desenvolvido pela própria equipe de pesquisa e contém atividades padrões (para todos os participantes), que exploram áreas como: motricidade, cognição, linguagem, sociabilidade, interação musical e estado emocional. Mesmo sendo padronizadas, as atividades exigiram certa flexibilidade para serem adaptadas às limitações de cada paciente, quando necessário. A criação dessas atividades foi baseada nos comportamentos observáveis pelas escalas IMTAP (The Individualized Music Therapy Assessment Profile) e SEMPA (Sistema de Evaluación Musicoterapéutica para Personas con Alzheimer $y$ otras Demencias) (INGELMO, 2012), que são escalas específicas da Musicoterapia. Além desses instrumentos, foram utilizados testes tradicionais realizados pela equipe médica da instituição, sendo eles: Mini Exame do Estado Mental - que avalia Orientação, Atenção e Cálculo, Memória imediata, Memória evocativa e Linguagem; teste do Relógio - que consiste em avaliar a cognição; Avaliação de Capacidade Funcional através das Escalas de Katz e Pfeffer; e por fim o Índice de Vulnerabilidade Clínico-Funcional (IVCF) que avalia idade, autopercepção de saúde, atividades de vida diária, cognição, humor, mobilidade, comunicação e comorbidades.

No presente artigo serão apresentadas comparações entre as avaliações inicial e final da escala SEMPA, para ambos os grupos de pesquisa. A escala SEMPA visa contemplar as áreas de atuações mais possíveis de serem trabalhadas em sessões de Musicoterapia com idosos (INGELMO, 2012, p. 70). A escala foi criada para auxiliar no desenvolvimento e manutenção das capacidades de pessoas com demências, podendo ser usada não apenas como método de avaliação em pesquisas, mas também como método de evolução do paciente após cada sessão para detecção de possíveis áreas a serem contempladas no processo terapêutico ${ }^{2}$. É possível

\footnotetext{
${ }^{2}$ Ibidem.
} 
observar através da escala as seguintes áreas: Psicomotriz, Perceptivo-Cognitivo, Comunicação-Linguagem e Socioemocional, em mensuração diretamente proporcional ao desempenho do participante. A partir dessas grandes áreas foram selecionados itens relevantes para a avaliação da pesquisa que estão expostos no quadro anexo (Anexo 1). A escala já foi usada em pessoas também sem demência e foi escolhida pela falta de escalas musicoterapêuticas na avaliação do idoso no Brasil (ZMITROWICZAB/ MOURA, 2018).

Para esta análise das avaliações inicial e final da SEMPA, foram utilizados os programas Microsoft Excel e Planilhas Google. A mensuração das avaliações foi feita por porcentagem para não contabilizar com valor 0 (zero) os itens que não puderem ser observados durante as avaliações. A comparação entre avaliações e entre grupos se deu por meio da comparação quantitativa, não estatística, das médias simples.

Durante as análises cega dos vídeos das avaliações iniciais e finais, além da aplicação da escala SEMPA, a pesquisadora também registrou comentários verbais dos participantes, que foram utilizados na presente pesquisa para reforçar ou refutar os resultados observados através da escala SEMPA.

\section{Resultados e discussão}

\subsection{Caracterização da amostra}

Dos 50 idosos selecionados para a pesquisa, 41 fizeram a avaliação inicial completa e 9 não conseguiram completar a avaliação, se recusaram a participar da pesquisa ou não puderam participar por motivo de internação. Durante o decorrer da pesquisa, 8 desistiram ou não puderam continuar também por motivo de internação ou óbito. Assim, restaram 33 idosos para análise da pesquisa, dentre eles 17 do grupo Intervenção e 16 do grupo Controle.

A idade dos idosos do grupo Intervenção variou de 78 a 95 anos (média 86,82), enquanto do grupo Controle variou de 78 a 96 anos (média 88,75), conforme pode ser observado no Quadro 2. Pode-se observar que as idades eram semelhantes entre os grupos. 
Quadro 2 - Caracterização de idade dos participantes por grupo.

\begin{tabular}{l|c|c}
\cline { 2 - 3 } & GRUPO INTERVENÇÃO & GRUPO CONTROLE \\
\hline IDADE MÍNIMA & 78 & 75 \\
\hline IDADE MÁXIMA & 95 & 96 \\
\hline MÉDIA & 86,30 & 87,47 \\
\hline
\end{tabular}

Elaboração da Autora.

O grupo Intervenção teve mais incidências de Doenças Cardíacas, seguida por Doenças pulmonares e dificuldades motoras e por último outras doenças fisiológicas. O grupo Controle também apresentou doenças cardíacas como principal diagnóstico, seguido por outras doenças fisiológicas, dificuldades motoras, demência e transtornos de humor. Conforme pode ser visto no quadro 3, podemos dizer que os grupos foram semelhantes entre si também em relação ao diagnóstico dos participantes.

\begin{tabular}{l|c|c}
\multicolumn{2}{c}{ Quadro 3 - caracterização de diagnóstico dos participantes por grupo. } \\
\cline { 2 - 3 } & GRUPO INTERVENÇÃO & GRUPO CONTROLE \\
\hline Doenças cardíacas & 10 & 9 \\
\hline Demências & 1 & 1 \\
\hline Dificuldades Motoras & 2 & 2 \\
\hline Doenças Pulmonares & & 1 \\
\hline Transtornos de Humor & 4 & 3 \\
\hline Outras doenças Fisiológicas & & \\
\hline
\end{tabular}

Elaboração da Autora.

\subsection{Comparação das avaliações iniciais e finais}

Ambos os grupos apresentaram, de uma maneira geral, a avaliação inicial melhor do que a avaliação final, considerando a média do Total da SEMPA. O Grupo Intervenção iniciou as médias com 84,97 (numa escala onde 100 era a máxima referência) e terminou com 80,79 (Gráfico 1) apresentando uma queda de 4,92\%. Já no Grupo Controle a queda foi de 9,35\%, tendo como média inicial 83,89 e final 76,05 (Gráfico 2). 
Gráfico 1 - Total SEMPA Intervenção.

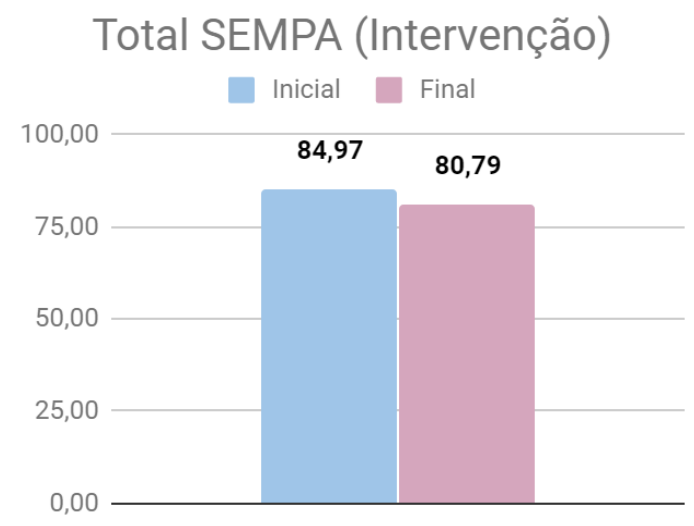

Gráfico 2 - Total SEMPA Controle.

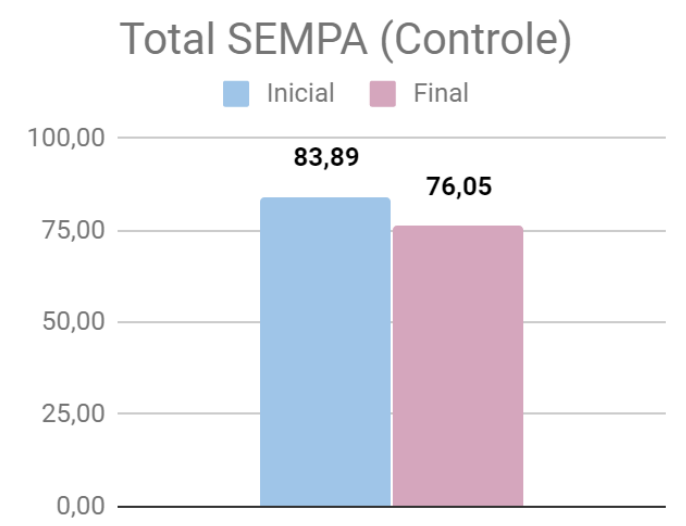

Quando avaliamos separadamente as áreas da SEMPA podemos notar que, no Grupo Intervenção, a área Psicomotriz teve uma queda de 5,0\% (de 78,47 para 74,53) e a CognitivoPerceptiva teve queda de 5,8\% (de 86,48 para 81,49). A área de Comunicação-Linguagem foi a que obteve a menor queda, sendo ela 2,1\% (de 85,32 para 83,50), e a área Socioemocional foi a que obteve a maior queda, de $7,2 \%(90,37$ para 83,83$)$. 
Gráfico 3: Avaliação de Áreas da SEMPA - Intervenção.

\section{Avaliação de Áreas da SEMPA}

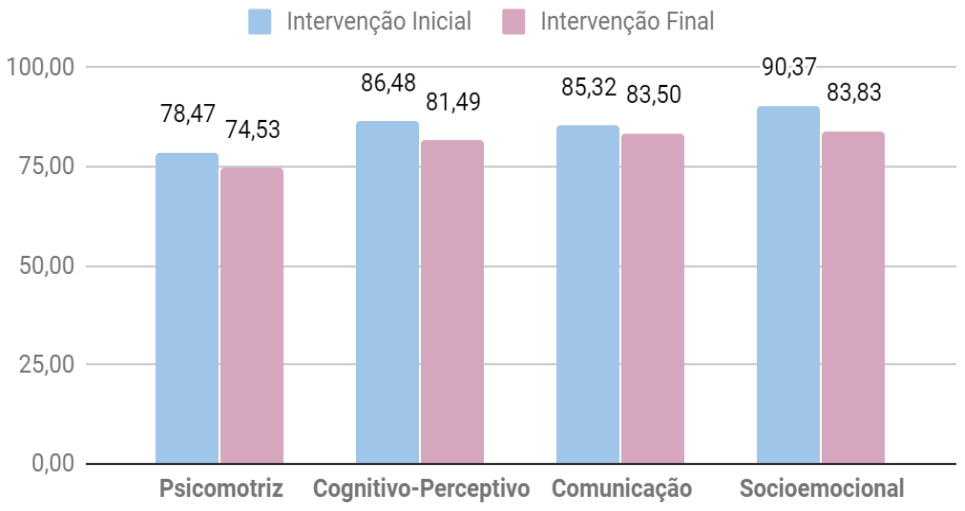

Fazendo essa mesma análise do grupo Controle, observamos que também houve declínio em todas as áreas avaliadas. Na área Psicomotriz houve o declínio de 4,5\% (de 73,28 para 70), na Cognitivo-Perceptiva de 12,2\% (de 84,48 para 74,15), em Comunicação 7,3\% (de 86,65 para 80,32) e na área Socioemocional de 8,3\% (de 89,44 para 82,03). As diferenças entre avaliações das áreas da SEMPA no grupo Controle podem ser vistas no Gráfico 4.

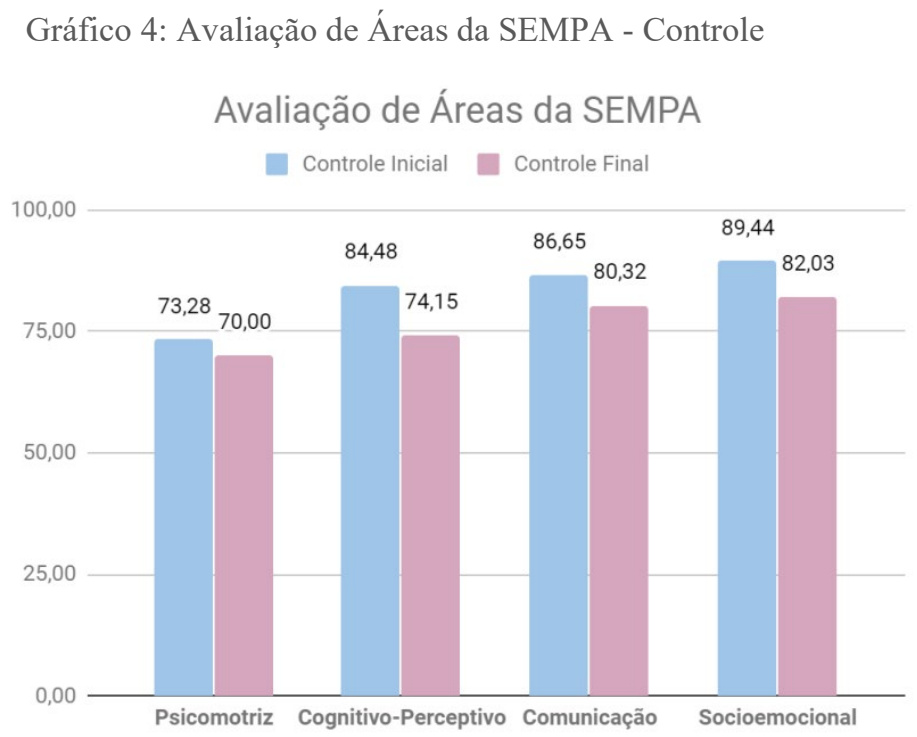

Podemos notar que os grupos iniciais (Intervenção e Controle) apresentam números semelhantes em todas as áreas de avaliação. Destacamos aqui que no grupo Controle as quedas são quase sempre maiores que no grupo Intervenção, com exceção da área Psicomotriz. 
Podemos perceber que a diferença mais relevante entre os grupos foi na área PerceptivoCognitivo, na qual o grupo Controle apresentou um déficit mais significativo (12,2\%).

Considerando a faixa etária dos grupos avaliados nessa pesquisa, podemos supor que haveria uma queda nas capacidades dos participantes devido ao processo de envelhecimento natural. Os resultados podem indicar que as intervenções musicoterapêuticas poderiam ser usadas para retardar esse processo nas áreas Cognitivo-Perceptivo, Comunicacional e Socioemocional do idoso.

Futuras análises serão realizadas pela equipe de pesquisa para verificar se há diferenças numericamente significativas, mostradas em testes estatísticos, entre essas quedas de pontuação nas avaliações iniciais e finais e entre os dois grupos. Serão realizadas também análises estatísticas com os dados dos outros instrumentos de avaliação citados na metodologia, que podem confirmar ou refutar os resultados encontrados até este momento.

Por outro lado, em observação qualitativa realizada durante as avaliações, foi notado que os idosos consideraram a experiência gratificante, o que em si já era um dos objetivos das sessões musicoterapêuticas: auxiliar no bem-estar diário. A experiência musical foi agradecida e engrandecida em muitas das avaliações. Muitos dos indivíduos exaltaram a presença do musicoterapeuta, dizendo que a música contribuiu de forma positiva para seu humor. Dessa forma, podemos inferir que as intervenções musicoterapêuticas puderam trazer resultados positivos aos participantes (resultados não mensurados pela escala), por confirmar a teoria de que a Musicoterapia tem papel de auxiliar no humor e no envelhecimento ativo (COLL, 2014).

Faz-se necessário também discutir pontos que podem ser considerados desafios na avaliação cega da escala. O primeiro ponto é que as quedas apresentadas nos grupos nem sempre significam que o paciente está mais debilitado. Alguns pacientes haviam acabado de passar por uma internação pouco antes de sua avaliação final, outros não estavam dispostos para realizar todas as atividades porque se diziam tristes por ser o último dia de interação com a musicoterapeuta avaliadora.

O segundo ponto diz respeito ao protocolo de atividade para avaliação, que era flexível para se adaptar às limitações de cada paciente, e apresentou maior adaptação individualizada na avaliação final. Assim, na avaliação final, não foi possível pontuar fielmente todos os comportamentos do idoso através da escala e, mesmo tendo ciência que o paciente conseguiria realizar o ponto a ser examinado, se não foi realizado na avaliação final, o item precisou ser desconsiderado. Essa diferença entre algumas atividades feitas nas avaliações inicial e final e a 
dificuldade de direcionamento dessas atividades para os itens da SEMPA a serem avaliados podem ter contribuído para a redução das pontuações nas avaliações finais.

Por fim, a forma de mensuração das avaliações, por porcentagem, desconsidera itens que não foram avaliados, exatamente para seguir a flexibilidade do protocolo. Ao usar essa lógica, os participantes que realizaram menos atividades tiveram mais chances de obter uma nota menor, pois cada atividade teria um peso maior e portanto, no caso do paciente não conseguir realizar algum item proposto, ele perderia mais pontos. Sugerimos que seja feita uma revisão no protocolo de atividades para que os itens possam ser mais assertivos e assim contemplar de maneira mais eficiente a aplicação avaliativa da escala SEMPA.

\section{Considerações Finais}

Neste artigo foram apresentados os resultados iniciais de uma pesquisa realizada com idosos em Musicoterapia domiciliar. O intuito foi avaliar os benefícios que a Musicoterapia, juntamente com um programa específico de atendimentos com materiais de apoio em vídeo, poderia promover para esses idosos.

Em razão das baixas pontuações obtidas na avaliação final, e apesar dos resultados apresentados no grupo Intervenção serem quantitativamente melhor do que o grupo Controle em três das quatro áreas avaliadas, não podemos afirmar se o programa de sessões utilizado é efetivo. Mais pesquisas precisam ser feitas para uma melhor adaptação das escalas de avaliação e dos protocolos de atividade, e para, assim, verificar melhor os efeitos das intervenções musicoterapêuticas nos idosos.

Ressaltamos a importância de novas pesquisas com outras avaliações e outros participantes, para demonstrar os benefícios que a Musicoterapia domiciliar pode trazer em prol da qualidade de vida e bem-estar do idoso. Ressaltamos também a seriedade da pesquisa em trazer fielmente os resultados e assim contribuir para o rigor científico em Musicoterapia, auxiliando também outros pesquisadores da área a refletirem sobre protocolos de avaliação e formas de mensuração.

\section{Agradecimentos}

Agradecemos aos musicoterapeutas Cláudia Miranda, Marina Reis e Yuri Pinheiro, por participarem desse projeto elaborando os protocolos de atendimento, materiais de apoio 
musicoterapêutico e realizando as sessões presenciais. Agradecemos também a CAPTAMED nas pessoas de Sheila Lopes, Camila Pimenta Guimarães, Ana Paula Ribeiro e Vanúbia Cordeiro, por participarem desse projeto conduzindo a parte administrativa, logística de convocação e contato com os pacientes, enfim, pela parceria institucional nesta pesquisa.

\section{Referências}

BARCELLOS, Lia Rejane Mendes. Diálogo entre as novas práticas da musicoterapia e os cursos de formação de musicoterapeutas. In: DREHER, S. C.; MAYER, G. C. T. A Clínica na Musicoterapia: avanços e perspectivas. São Leopoldo: EST, 2014. p. 61-75.

CEBA, Maria del Mar Ordõno; BARÓN, Aida Fernández; RODRÍGUEZ, Vanesa Fernández. Beneficios de la musicoterapia en ancianos con demencia. In: PÉREZ-FUENTES, Maria del Carmen Cuidados, aspectos psicológicos y actividad física en relación con la salud Volumen II, 2016. Cap. 52, p. 355-360.

COLL, Orion Casals. Música, Reminiscencia y Envejecimiento Activo. In: Congreso Nacional de Musicoterapia. 5 ed. 2014. Barcelona. Orquestrando la Musicoterapia. p. 137-138.

INGELMO, Maria Elena González (Org.). SEMPA: Sistema de Evaluación Musicoterapéutica para Personas con Alzheimer y otras Demencias. 1 ed. Madrid: Ministerio de Sanidad, Servicios Sociales e Igualdad. 2012. NIPO: 686-12-011-0.

KARST, Lara T. A musicoterapia na assistência domiciliar aos cuidadores da criança em cuidados paliativos oncológicos. Goiânia, 2015. Dissertação (Mestrado em Música) - Universidade Federal de Goiás, Escola de Música e Artes Cênicas (Emac), Programa de Pós-Graduação em Música, Goiânia, 2015.

MARQUES, Daiane Pazzini. A importância da musicoterapia para o envelhecimento ativo. Revista Portal de Divulgação, v. 15, p. 18-24, 2011.

PINHEIRO, Y.; REIS, M.; MIRANDA, C.; BARBOSA, S.; FREIRE, M. Musicoterapia na assistência domiciliar a idosos. Revista Brasileira de Musicoterapia, ano XIX, ed. especial, p. 266-269, 2017.

SEQUERA-MARTÍN, et al. Musicoterapia en la demencia del paciente anciano: fundamentos, aplicaciones y evidencia científica actual. Psicogeriatría, Espanha, v. 5, p. 93-100, 2015.

WFMT - WORLD FEDERATION OF MUSIC THERAPY. What is Music Therapy?, 2011. Disponível em: <http://www.wfmt.info/wfmt-new-home/about-wfmt/>. Acesso em: 12 jul. 2021.

ZMITROWICZAB, J.; MOURA, R. Instrumento de avaliação em Musicoterapia: uma revisão. Revista Brasileira de Musicoterapia, v. XX, n. 24, p. 114-135, 2018. 


\section{Anexo 1}

Itens da SEMPA (INGELMO, 2012) avaliados nesta pesquisa, de acordo com o protocolo aplicado.

\begin{tabular}{|c|c|}
\hline PSICOMOTRIZ & PERCEPTIVO-COGNITIVO \\
\hline $\begin{array}{l}\text { Capaz de explorar instrumentos com os dedos } \\
\text { das mãos }\end{array}$ & $\begin{array}{l}\text { Capaz de decidir entre duas ou mais alternativas oferecidas } \\
\text { como solução a um determinado problema }\end{array}$ \\
\hline $\begin{array}{l}\text { Capaz de fazer percussão corporal com } \\
\text { diferentes partes do corpo }\end{array}$ & Capaz de responder a estímulos auditivos \\
\hline $\begin{array}{l}\text { Capaz de segurar um instrumento que o } \\
\text { musicoterapeuta coloca em suas mãos }\end{array}$ & Capaz de localizar de maneira auditiva uma fonte sonora \\
\hline $\begin{array}{l}\text { É capaz de se manter em equilíbrio enquanto } \\
\text { está sentado }\end{array}$ & Capaz de diferenciar 2 ou mais sons diferentes \\
\hline $\begin{array}{l}\text { É capaz de se manter em equilíbrio durante a } \\
\text { marcha }\end{array}$ & Realiza uma ação ao escutar um estímulo sonoro \\
\hline Ativa o corpo a partir de estímulos musicais & Capaz de planejar diversas ações diárias \\
\hline $\begin{array}{l}\text { Capaz de se manter em equilíbrio enquanto está } \\
\text { em pé }\end{array}$ & Capaz de memorizar padrões rítmico-melódicos \\
\hline $\begin{array}{l}\text { Capaz de mover partes do corpo quando } \\
\text { requisitado }\end{array}$ & $\begin{array}{l}\text { Capaz de reconhecer ao menos uma música escutada no } \\
\text { passado }\end{array}$ \\
\hline $\begin{array}{l}\text { Capaz de usar a preensão de pinça das mãos } \\
\text { para segurar um objeto }\end{array}$ & Capaz de mencionar canções, descrevendo suas características \\
\hline $\begin{array}{l}\text { Capaz de soltar um instrumento para pegar o } \\
\text { outro }\end{array}$ & Capaz de cantar letras de canções conhecidas \\
\hline $\begin{array}{l}\text { Adapta pulso rítmico de acordo com } \\
\text { modificações rítmicas na atividade }\end{array}$ & Capaz de manter a atenção durante as atividades musicais \\
\hline \multirow[t]{8}{*}{ Marca pulso rítmico de maneira intencional } & $\begin{array}{l}\text { Capaz de se lembrar de episódios importantes da vida a pedido } \\
\text { do musicoterapeuta }\end{array}$ \\
\hline & $\begin{array}{l}\text { Mantém a atenção na conversa olhando para quem está } \\
\text { falando }\end{array}$ \\
\hline & Capaz de responder a estímulos visuais \\
\hline & Capaz de localizar visualmente um estímulo \\
\hline & Capaz de responder quando é chamado pelo nome \\
\hline & Capaz de dizer o ano/mês/semana/dia que se encontra \\
\hline & Capaz de se lembrar a da data de nascimento \\
\hline & Ao final da sessão é capaz de recordar uma atividade realizada \\
\hline
\end{tabular}


Anexo 2

\begin{tabular}{l|l}
\hline \multicolumn{1}{c|}{ COMUNICAÇÃO E LINGUAGEM } & \multicolumn{1}{c}{ SOCIOEMOCIONAL } \\
\hline $\begin{array}{l}\text { Compreende ordem dada pelo } \\
\text { musicoterapeuta }\end{array}$ & Mostra atitude de ajuda e colaboração com outras pessoas \\
\hline $\begin{array}{l}\text { Capaz de emitir sons vocais em um contexto } \\
\text { comunicativo com o musicoterapeuta }\end{array}$ & $\begin{array}{l}\text { Faz comentários positivos sobre as tarefas e/ou sobre o(a) } \\
\text { musicoterapeuta }\end{array}$ \\
\hline Imita sons não verbais & Mostra atitude de escuta \\
\hline Se expressa oralmente & Capaz de expressar humor \\
\hline Imita movimentos corporais & Capaz de expressar emoções \\
\hline Se expressa corporalmente para se comunicar & Capaz de falar de si mesmo \\
\hline Mantém jogo de turnos com outra pessoa & Realiza a atividade porque tem interesse e curiosidade \\
\hline Compreende a letra das canções & Capaz de ter uma valorização positiva de sua personalidade \\
\hline $\begin{array}{l}\text { Estabelece com naturalidade um discurso } \\
\text { coerente com boa coesão }\end{array}$ & Capaz de expressar agradecimento \\
\hline $\begin{array}{l}\text { Explica e expressa opiniões sobre uma } \\
\text { canção escolhida }\end{array}$ & $\begin{array}{l}\text { Expressa satisfação ou realiza comentários positivos sobre si } \\
\text { mesmo por uma ou mais tarefas realizadas previamente }\end{array}$ \\
\hline Comunicação e Linguagem & Socioemocional \\
\hline
\end{tabular}

